EXTENDED REPORT

\title{
Cancer incidence among patients with ankylosing spondylitis in Sweden 1965-95: a population based cohort study
}

\author{
N Feltelius, A Ekbom, P Blomqvist
}

Ann Rheum Dis 2003;62:1185-1188. doi: 10.1136/ard.2002.004721

See end of article for authors' affiliations .....................

Correspondence to: Dr N Feltelius, Medical Products Agency, PO Box 26, SE-751 03 Uppsala, Sweden; Nils.Feltelius@mpa.se

Accepted 22 January 2003

.....................
Background: Ankylosing spondylitis (AS) is a chronic inflammatory joint disease which may lead to extraarticular complications. The disease associated cancer risk has been poorly explored. Recently, tumour necrosis factor $\alpha$ blockers have been found to be efficacious in AS, but their long term risk is unknown. Objective: To analyse a large national cohort of patients with AS to determine the overall cancer risk in AS as a background to the future introduction of new treatments.

Methods: All patients with AS admitted to Swedish hospitals 1965-95 were linked through individual national registration numbers to the Swedish Cancer Register and National Death Register. Standardised incidence ratio (SIR) of cancer risk was calculated in 6621 people, monitored during 67885 personyears.

Results: No overall increase in cancer risk was found (SIR 1.05, 95\% Cl 0.94 to 1.17). Rectal cancer was less common (SIR $0.41,95 \% \mathrm{Cl} 0.15$ to 0.89 ) while unspecified kidney cancer was more common (SIR $5.90,95 \% \mathrm{Cl} 1.61$ to 15.1$)$. Risks for colon, renal parenchymal, and renal pelvic cancer were not significantly increased. Laryngeal cancer was more common than expected, while lung cancer was not. Risks of haematopoietic malignancies were not increased.

Conclusions: No overall increase in cancer risk was found. The decreased risk of rectal cancer might be due to local application of NSAIDs, and the increased risk of unspecified kidney cancer to frequent radiological pelvic examinations. If information on disease characteristics, including HLA-B27, was available for individual patients with cancer, risk-benefit analysis of long term effects of new immunomodulation treatment might be improved.
$\mathrm{T}$ he role of chronic inflammation in the development of cancer has been a topic of both epidemiological and experimental studies. In rheumatoid arthritis (RA), an increased risk of lymphoma has been reported. ${ }^{1}$ Treatment with cytotoxic drugs has been linked to the development of specific cancers-for example, cyclophosphamide and bladder cancer. ${ }^{2}$ Chronic infection or persistent inflammation has been suggested as a driving force for de novo lymphogenesis, resulting in malignancy. ${ }^{3}$ High inflammatory activity has been proposed as an independent risk factor for lymphomas in patients with RA. ${ }^{4}$ In ulcerative colitis an increased risk of colorectal cancer has been firmly linked to extensive longstanding disease. ${ }^{5}$ In other organs, such as the lung and liver, chronic inflammation, sometimes in connection with fibrosis, is associated with malignant transformation. ${ }^{6}$

Although ankylosing spondylitis (AS) shares the basic characteristics of a chronic inflammatory joint disease with $\mathrm{RA}$, there are also clear differences. The sex distribution is different, with a male predominance in AS, and the joint involvement in AS is mainly axial, compared with peripheral in RA. The inflammatory activity is often lower in AS and the pattern of extra-articular manifestations differs. ${ }^{7}$ Also, the high prevalence of HLA-B27 and its subtypes in patients with AS is a unique characteristic. ${ }^{8}{ }^{9}$

The increased cancer risk in patients with AS subjected to radiation treatment has been extensively studied. ${ }^{10}$ In Sweden, the use of this treatment for locomotor diseases has been very limited, and during the past decades nonexistent. ${ }^{11}$

Immunomodulating agents have hitherto been less widely used in AS than in RA. ${ }^{12}$ Promising results of tumour necrosis factor blockade in AS have recently been published and this may alter the situation. ${ }^{13}$ However, the effects of long term inhibition of cytokines, including the possibility of an increased risk of cancer, are unknown. With the emergence of this new therapeutic potential, a study describing the overall cancer risk in a well characterised AS population can provide valuable information. To our knowledge, no studies have examined the overall cancer risk in a large cohort of "radiation-naive" patients with AS, derived from national population based registries.

\section{METHODS}

\section{The cohort}

In 1965 the National Board of Health and Welfare started collecting data on hospital discharges in the Inpatient Register. The coverage expanded and in 1983, information on $85 \%$ of all annual admissions was collected. Since 1987, all public hospitals have participated in this register. The records contain some 30 variables characterising the patient, the admission, diagnoses, operations or procedures, and hospital information. Each patient is identified by a national registration number (NRN), unique for every Swedish resident. Validation of the Inpatient Register has shown that it has a satisfactory level of accuracy. ${ }^{14}$ Because private inpatient care in Sweden is rare, most patients are admitted to public hospitals in the county where they reside. Thus, the

Abbreviations: AS, ankylosing spondylitis; $\mathrm{Cl}$, confidence interval; $\mathrm{Gl}$, gastrointestinal; NRN, national registration number; NSAIDs, nonsteroidal anti-inflammatory drugs; RA, rheumatoid arthritis; SIR, standardised incidence ratio 
Inpatient Register essentially reflects the morbidity of the population of the different counties.

From the Inpatient Register for 1965-95, we selected all records concerning patients with a primary or secondary diagnosis of AS (ICD-7: 722.1, ICD-8: 712.4, ICD-9:720A). Because the Inpatient Register records discharge data and not individuals, cross linkage within the register identified the first discharge (index admission to hospital) with this disease for each affected person. This procedure yielded 8345 people with one or several admission records indicating AS.

For each person the data from the Inpatient Registry were then examined and several exclusions followed (fig 1). First, all patients not only having AS but also having been admitted earlier for RA or psoriasis were excluded ( $\mathrm{n}=850 ; 10.2 \%)$. In addition, every individual NRN was corroborated against the Total Population Register, the National Migration Register, and the National Death Register, all maintained by Statistics Sweden. Some inconsistencies, for example internal missing data, were discovered and a total of 577 (6.9\%) were further excluded. This left 6918 patients of the original study cohort, eligible for cancer follow up. Among these, 75.3\% were men. The median age was 43 years (quartile range 33-56).

\section{Follow up}

To ascertain the risk of cancer, records of these patients with AS were linked to the Swedish Cancer Register..$^{15}$ In addition, linkage to the National Death Register, which provides information on the date and causes of death was performed. All national health registries are maintained by the National Board of Health and Welfare and have a high validity. ${ }^{16-19}$ To avoid ascertainment bias related to differences in necropsy rates over time and between geographical areas among patients with AS and the general population, we also excluded cancers diagnosed incidentally at necropsy from both the observed and expected rates. We only included the first cancer of each person.

This linkage initially yielded 407 people with tumours. To minimise the impact of cancers that were the underlying reason for admission to hospital, and a potential selection bias resulting from an increased likelihood of cancer detection during admission to hospital, we excluded all first year tumours $(\mathrm{n}=82)$ from further analysis. This left 325

\begin{tabular}{|lcc|}
\hline & No & $\%$ \\
\hline $\begin{array}{l}\text { Ratients with AS } \\
\text { Rheumatoid arthritis } \\
\text { or psoriasis with AS }\end{array}$ & 8345 & 100.0 \\
$\begin{array}{l}\text { Inconsistent data } \\
\begin{array}{l}\text { Cancer detected first year } \\
\text { or patients monitored less } \\
\text { than one year }\end{array}\end{array}$ & $\frac{7495}{677}$ & 89.8 \\
& $\frac{6918}{297}$ & 82.9 \\
\hline
\end{tabular}

Figure 1 Cancer incidence among patients with AS in Sweden 1965-95. Characteristics of the study base. tumours registered one year or later after the first AS admission.

\section{Statistical methods}

As a measure of the risk of patients with AS developing tumours, the standardised incidence ratio (SIR) was calculated. ${ }^{20}$ This was defined as the ratio of observed number of cancers to the expected number, taking into consideration the person-years at risk. The time of observation for the patients with AS was calculated from the date of their first AS discharge to the occurrence of a tumour, death, emigration, or end of follow up on 31 December 1995. To correspond with exclusion of tumours detected in the first year, the first year of follow up was also excluded. This left 6621 people who were monitored during a total of 67885 person-years.

The expected number of tumours was calculated on the basis of sex, calendar year, and five year age group specific cancer incidence rates derived from the Swedish population. A 95\% confidence interval (CI) of SIR was calculated on the assumption that the observed findings followed a Poisson distribution. ${ }^{20}$ We also characterised each patient by age at entry, attained age when developing cancer, follow up years, and time period.

\section{RESULTS}

During the first year of follow up of the patients with AS, 82 tumours were reported, $45(55 \%)$ of these within three months after the admission. The patients' median age was 64 years (quartile range 54-72). Table 1 presents the diagnoses. Digestive tract cancers were common (20.7\%) and were mainly stomach and colorectal cancers. Also common were prostate cancer (18.3\%) and haematopoietic cancers (14.6\%).

We identified 325 patients in whom a first cancer was registered after the first year of follow up (table 2). There was no overall increase in the risk of developing tumours among patients with AS (SIR 1.05, 95\% CI 0.94 to 1.17). Adjustments for the patients' sex, age at entry, attained age at cancer diagnosis, and follow up years, all yielded the same results. SIR for the overall risk was increased during first time period, 1965-75, but not during later periods.

In table 3, SIR for each cancer type is presented. No cancer group had an SIR that differed significantly from the expected rates. Each organ group was also analysed in detail for individual tumours. One significant finding was that rectal cancer (ICD-7: 154) was less common than in the general population (SIR $0.41,95 \%$ CI 0.15 to 0.89 ). This was noted in both men and women, and also, though without reaching the level of significance, in all time periods, and decreased with follow up time. A lower incidence of colon cancer was not detected (ICD-7: 153) (SIR 0.95, 95\% CI 0.58

Table 1 Cancer incidence among patients with AS in Sweden 1965-95. Number (\%) reported during the first year of follow up

\begin{tabular}{lrr}
\hline Diagnosis (ICD-7) & No & $\%$ \\
\hline Digestive cancer (150-159) & 17 & 20.7 \\
Respiratory cancer (160-164) & 9 & 11.0 \\
Breast cancer (170) & 3 & 3.7 \\
Prostate cancer (177) & 15 & 18.3 \\
Kidney cancer (180) & 3 & 3.7 \\
Cancer of urinary organs (181) & 7 & 8.5 \\
Cancer of nervous system (193) & 7 & 8.5 \\
Other cancers (194-199) & 9 & 11.0 \\
All haematopoietic cancers (200-209) & 12 & 14.6 \\
All cancers & 82 & 100.0 \\
\hline
\end{tabular}


Table 2 Cancer incidence among patients with AS in Sweden 1965-95

\begin{tabular}{lccl}
\hline & $\begin{array}{c}\text { Observed } \\
\text { cancers }\end{array}$ & SIR & $95 \% \mathrm{Cl}$ \\
\hline $\begin{array}{l}\text { Sex } \\
\text { Male }\end{array}$ & 262 & 1.08 & 0.96 to 1.22 \\
$\begin{array}{l}\text { Female } \\
\text { Age at entry }\end{array}$ & 63 & 0.92 & 0.71 to 1.18 \\
0-19 & 2 & 2.42 & 0.29 to 8.73 \\
$20-39$ & 56 & 1.17 & 0.88 to 1.51 \\
$40-59$ & 158 & 1.02 & 0.86 to 1.19 \\
60- & 109 & 1.03 & 0.84 to 1.24 \\
Attained age at cancer diagnosis (years) & & \\
$0-19$ & 0 & - & - \\
$20-39$ & 18 & 1.47 & 0.87 to 2.33 \\
$40-44$ & 15 & 1.17 & 0.66 to 1.94 \\
$45-49$ & 15 & 0.74 & 0.42 to 1.23 \\
$50-59$ & 67 & 1.12 & 0.86 to 1.42 \\
$60-$ & 210 & 1.02 & 0.89 to 1.17 \\
Follow up time (years) & & & \\
$0-5$ & 92 & 1.02 & 0.82 to 1.25 \\
6-10 & 81 & 0.91 & 0.72 to 1.13 \\
$11-15$ & 74 & 1.14 & 0.89 to 1.43 \\
$16-$ & 78 & 1.18 & 0.93 to 1.47 \\
Time period & & & \\
$1965-75$ & 137 & 1.22 & 1.03 to 1.45 \\
$1976-85$ & 129 & 0.90 & 0.75 to 1.07 \\
$1986-95$ & 59 & 1.07 & 0.82 to 1.38 \\
All cancers & 325 & 1.05 & 0.94 to 1.17 \\
\hline & & &
\end{tabular}

to 1.47 ), nor of stomach cancer (ICD-7: 151) (SIR 0.95, 95\% CI 0.51 to 1.63 ).

Both melanoma and non-melanoma skin cancers were less common, but not significantly (ICD-7: 190, SIR 0.72; ICD-7: 191 SIR 0.76, respectively). Among respiratory tract cancers, the risk of developing laryngeal cancer (ICD-7: 161) was increased (SIR 2.49, 95\% CI 1.00 to 5.12). This risk was particularly high in the early years of the study period and increased with follow up time. The risk of lung cancer (ICD-7: 162-163) was not increased (SIR 0.93 NS).

Unspecified kidney cancer (ICD-7: 180.9) was significantly more common (SIR 5.90, 95\% CI 1.61 to 15.1 ). This risk was increased in all time periods. Within $1-5$ and $5-10$ years of follow up it was increased fivefold (SIR 5.5 NS; SIR 5.6; NS).
After 10-15 years it increased further (SIR 13.89, 95\% CI 1.68 to 50.2). Both renal parenchyma and renal pelvic cancer were also more common, though not significantly.

The risk of cancer of the nervous system (ICD-7 193) was increased (SIR 1.34 NS). This was found both among men and women and in all age groups. The increased rate was significant during the last time period, 1985-95 (SIR 3.55, $95 \%$ CI 1.43 to 7.32 ), and within the first five years of follow up (SIR 2.57, 95\% CI 1.11 to 5.06).

There was no significantly increased risk of any haematopoietic malignancy. However, all leukaemias combined showed an increased SIR, but the increase was not significant (ICD-7 204-207: SIR 1.59 NS).

\section{DISCUSSION}

The main finding in our study is that there is no overall increase in cancer risk in patients with AS. The most striking result relating to a specific organ system is the decreased risk of rectal cancer without a similar reduction in the risk of other gastrointestinal (GI) cancers. Minor increases in some other cancer forms were seen.

When cancers detected during the first year of admission were analysed separately, a high rate of prostatic cancer (18.3\%) was noted. However, this seems to be well compatible with the age (mean 64 years) and sex (70\% male) distribution of the cohort. ${ }^{21}$ No other unexpected findings were seen during this first year.

The analyses disclosed no generally increased risk of cancer in this cohort of patients with AS when consideration was paid to age, sex, age at entry, attained age at cancer diagnosis, follow up years, and time period. A slightly increased risk (SIR 1.22) in the first time period (1965-75) was found (table 2). There is no obvious explanation for this finding. Studies of patients with AS who have been exposed to therapeutic $x$ rays have shown significant increases in haematological cancers, such as multiple myeloma and several others, with a significant dose-response relationship for some of them. ${ }^{10}$ Possibly, the use of therapeutic radiation treatment for locomotor diseases during the 1950s might have influenced the cancer risk in our cohort a decade later. ${ }^{11}$ Since that time this treatment has not been used in Sweden.

The reason for the decreased risk of rectal cancer without a similar risk estimation for colon cancer or any other GI cancer

Table 3 Cancer incidence among patients with AS in Sweden 1965-95. Standardised incidence ratio (SIR) and corresponding $95 \%$ confidence interval. Cancers reported $\geqslant 1$ year after first admission with AS

\begin{tabular}{lccc}
\hline Diagnosis (ICD-7) & $\begin{array}{c}\text { Observed } \\
\text { cancers }\end{array}$ & SIR & 95\% Cl \\
\hline Buccal cancer (140-148) & 9 & 1.11 & 0.51 to 2.11 \\
Cancer of digestive organs (150-159) & 67 & 0.96 & 0.74 to 1.21 \\
Respiratory cancer (160-164) & 34 & 1.05 & 0.73 to 1.47 \\
Breast cancer (170) & 20 & 0.92 & 0.56 to 1.42 \\
Cancer of female genital system (171-176) & 11 & 0.94 & 0.47 to 1.68 \\
Prostate cancer (177) & 55 & 1.02 & 0.77 to 1.33 \\
Testicular and other male cancer (178-179) & 3 & 0.87 & 0.18 to 2.53 \\
Kidney cancer (180) & 16 & 1.54 & 0.88 to 2.51 \\
Cancer of urinary organs (181) & 20 & 1.05 & 0.64 to 1.62 \\
Melanoma skin cancer (190) & 9 & 0.72 & 0.33 to 1.37 \\
Non-melanoma skin cancer (191) & 8 & 0.76 & 0.33 to 1.50 \\
Eye cancer (192) & 2 & 2.26 & 0.27 to 8.16 \\
Cancer of nervous system (193) & 14 & 1.34 & 0.73 to 2.24 \\
Thyroid cancer (194) & 2 & 0.83 & 0.11 to 3.23 \\
Endocrine cancer (195) & 9 & 1.73 & 0.79 to 3.20 \\
Bone cancer (196) & 2 & 3.64 & 0.44 to 13.2 \\
Connective tissue cancer (197) & 2 & 0.88 & 0.11 to 3.19 \\
Other cancers & 9 & 0.93 & 0.43 to 1.77 \\
Haematopoietic cancer (200-208) & 33 & 1.34 & 0.93 to 1.89 \\
All cancers & 325 & 1.05 & 0.94 to 1.17 \\
\hline
\end{tabular}


is not known. In other studies, a decreased risk of GI cancer has been linked to the use of non-steroidal anti-inflammatory drugs (NSAIDs), both in clinical and experimental situations. ${ }^{22}{ }^{23}$ This effect has been attributed to the systemic effects of these drugs. ${ }^{24}$ Whether local application of NSAIDs, which was often used by patients with AS in Sweden in previous years, has an anticancer effect could not be settled by this study, but the possibility gains some support from experimental studies. ${ }^{25}$

Unspecified kidney cancer was more common than in the general population, as also was renal parenchymal and renal pelvic cancer. Other studies have shown that therapeutic $x$ ray examinations increase the risk for kidney cancers. ${ }^{10}$ Repeated irradiation exposure by frequent diagnostic $x$ ray examinations of the pelvis may explain these findings in our study.

There was no significant increase in any haematopoietic cancer. This is in clear contrast with results reported for patients with AS treated with irradiation. ${ }^{10}$ Of particular interest is the fact that no increase in lymphoma risk was seen. Baecklund et al suggested that lymphoma risk correlates with the inflammatory activity of the joint disease. ${ }^{4}$ Thus, lower disease activity in patients with AS compared with those with RA might explain a difference in lymphoma risk. For all leukaemias combined, SIR was increased. A possible decrease over time might be explained by decreased use of both therapeutic and diagnostic $x$ ray examinations.

In addition to disease severity, as reflected by inflammatory markers and the natural course of the disease, possible infectious agents as well as genetic factors (HLA-B27) need to be considered when assessing the cancer risk associated with AS. Persistent activation of the immune system by chronic infections has been suggested as a possible pathogenetic mechanism in AS, a theory which has gained some support from the beneficial treatment effects of antibacterial agents, like sulfasalazine in these patients. ${ }^{26-28}$ However, this concept has been criticised, and no specific bacterial or viral agent has yet been linked to the pathogenesis of AS. The hypothesis of a longstanding subclinical infection as a trigger of cancer in patients with AS does not find support in our study.

A limitation of our study is that the cohort consists of inpatients, defined by the primary diagnosis in patients admitted to hospital. Thus it does not represent the entire spectrum of disease prevalence, activity, complications, and drug exposure of patients with AS. On the other hand, it seems likely that the great majority of patients with AS in Sweden during our study period had been admitted at least once during the course of their disease, because the diagnostic management or complex drug treatment is seldom performed completely in an outpatient setting.

A particular strength of our study is its nationwide scope, the long observation period, and the fact that the data are derived from large, valid NRN based registries. Still we have no detailed information of individual disease severity, drug regimens or HLA characteristics of the patients who actually developed tumours because these data were not available in the Inpatient Registry.

In conclusion, we found no overall increase in cancer risk in patients with AS. At present the new biological agents, profoundly interfering with the immune system, are being introduced in the treatment of AS. In this situation our study provides a valuable reference of the cancer risk in AS, when evaluating patients' disease development and drug reactions.

\section{Authors' affiliations}

N Feltelius, Unit of Rheumatology, Department of Medicine at Karolinska Hospital, Karolinska Institutet, Stockholm, Sweden
A Ekbom, Unit of Clinical Epidemiology, Department of Medicine at Karolinska Hospital, Karolinska Institutet, Stockholm, Sweden

P Blomqvist, Unit of Clinical Epidemiology, Department of Medicine at Karolinska Hospital, and Department of Medical Epidemiology,

Karolinska Institutet, Stockholm, Sweden

\section{REFERENCES}

1 Gridley G, McLaughlin JK, Ekbom A, Klareskog L, Adami HO, Hacker DG, et al. Incidence of cancer among patients with rheumatoid arthritis. J Natl Cancer Inst 1993:85:307-11.

2 Radis CD, Kahl LE, Baker GL, Wasko MC, Cash JM, Gallatin A, et al. Effects of cyclophosphamide on the development of malignancy and on long-term survival of patients with rheumatoid arthritis. A 20-year followup study. Arthritis Rheum 1995:38:1120-7.

3 Hjelmstrom P. Lymphoid neogenesis: de novo formation of lymphoid tissue in chronic inflammation through expression of homing chemokines. J Leukoc Biol 2001;69:331-9.

4 Baecklund E, Ekbom A, Sparen P, Feltelius N, Klareskog L. Disease activity and risk of lymphoma in patients with rheumatoid arthritis: nested case-control study. BMJ 1998;317:180-1.

5 Ekbom A. Risk of cancer in ulcerative colitis. J Gastrointest Surg 1998:2:312-13.

6 Bouros D, Hatzakis K, Labrakis H, Zeibecoglou K. Association of malignancy with diseases causing interstitial pulmonary changes. Chest 2002;121:1278-89.

7 Kerr HE, Sturrock RD. Clinical aspects, outcome assessment, disease course, and extra-articular features of spondyloarthropathies. Curr Opin Rheumatol 1999;11:235-7.

8 Reveille JD, Ball EJ, Khan MA. HLA-B27 and genetic predisposing factors in spondyloarthropathies. Curr Opin Rheumatol 2001;13:265-72.

9 Sesma L, Montserrat V, Lamas JR, Marina A, Vasquez J, Lopez de Castro JA. The peptide repertoires of HLA-B27 subtypes differentially associated to spondylarthropathy $\left(B^{*} 2704\right.$ and $\left.B^{*} 2706\right)$ differ by specific changes at three anchor positions. Biol Chem 2002;277:16744-9.

10 Weiss HA, Darby SC, Doll R. Cancer mortality following x-ray treatment for ankylosing spondylitis. Int J Cancer 1994;59:327-38.

11 Jonson E. Indikationer för röntgenbehandling vid reumatiska sjukomar. Svenska Läkartidningen 1947;44:2104-13.

12 Dougados M, Dijkmans B, Khan M, Maksymowych W, Van Der Linden S Brand J. Conventional treatments for ankylosing spondylitis. Ann Rheum Dis 2002;61(suppl III):iii40-50.

13 Brandt J, Haibel H, Reddig J, Sieper J, Braun J. Successful short term treatment of severe undifferentiated spondyloarthropathy with the anti-tumor necrosis factor-alpha monoclonal antibody infliximab. J Rheumatol 2002;29:118-22.

14 Spetz CL, Carlsson CL, Engqvist M, Forsberg L, Aberg A, Akesson LO, et al. Reintroduction of social security numbers gives better basis for evaluation. The patient registry is open for research. Lakartidningen 1996;93:1844-7, [In Swedish].

15 Centre for Epidemiology. Cancer incidence in Sweden 1996. Centre for Epidemiology, Stockholm, Sweden: The National Board of Health and Welfare, 1998.

16 Centre for Epidemiology. Causes of death 1996. Centre for Epidemiology, Stockholm, Sweden: The National Board of Health and Welfare, 1998.

17 Nyrén O, McLaughlin JK, Gridley G, Ekbom A, Johnell O, Fraumeni JF Jr, et al. Cancer risk after hip replacement with metal implants: a populationbased cohort study in Sweden. J Natl Cancer Inst 1995:87:28-33.

18 de Faire $U$, Friberg $L$, Lorich $U$, Lundman T. A validation of cause of death certification in 1156 deaths. Acta Med Scand 1976;200:223-8.

19 Sundman L, Jakobsson S, Nyström L, Rosén M. A validation of cause of death certification for ischemic heart disease in two Swedish municipalities. Scand J Prim Health Care 1988:6:205-11.

20 Rothman KJ. Modern epidemiology, 2nd ed. Philadelphia: Lippincott-Raven, 1998.

21 Nomura AMY, Kolonel LN. Prostate cancer: a current perspective. Epidemiol Rev 1991;13:200-27.

22 Rosenberg L, Louik C, Shapiro S. Nonsteroidal antiinflammatory drug use and reduced risk of large bowel carcinoma. Cancer 1998;82:2326-33.

23 Thun MJ. Aspirin, NSAIDs and digestive tract cancers. Cancer Metastatis Rev 1994:13:269-77.

24 Shiff SJ, Koutsos MI, Qiao L, Rigas B. Nonsteroidal antiinflammatory drugs inhibit the proliferation of adenocarcinoma cells: effects on cell cycle and apoptosis. Exp Cell Res 1996;222:179-88.

25 Ligumsky M, Sestieri M, Karmeli F, Zimmerman J, Okon E, Rachmilewitz D. Rectal administration of nonsteroidal antiinflammatory drugs. Effect on rat gastric ulcerogenicity and prostaglandin E2 synthesis. Gastronterology 1990;98(Pt 1):1245-9.

26 Tiwana H, Wilson C, Walmsley RS, Wakefield AJ, Smith MS, Cox NL, et al. Antibody responses to gut bacteria in ankylosing spondylitis, rheumatoid arthritis, Crohn's disease and ulcerative colitis. Rheumatol Int 1997; 17:11-16.

27 Toivanen P, Hansen DS, Mestre F, Lehtonen L, Vaahtouvo J, Vehma M, et al Somatic serogroups, capsular types, and species of fecal Klebsiella in patients with ankylosing spondylitis. J Clin Microbiol 1999;37:2808-12.

28 Feltelius N, Hvatum M, Brandtsaeg P, Hallgren R. Increased jejunal secretory $\lg A$ and $\lg M$ in ankylosing spondylitis: normalization after treatment with sulfasalazine. J Rheumatol 1994;21:2076-81. 